\title{
Compreensão em Leitura em Estudantes do Terceiro e Quarto Ciclos do Ensino Fundamental
}

\author{
Adriana Cristina Boulhoça Suebiro - Universidade Federal do Recôncavo da Babia, Santo Antônio de Jesus, Brasil \\ Evely Boruchovitch - Universidade Estadual de Campinas, Campinas, Brasil
}

\begin{abstract}
Resumo
Este estudo teve por objetivos identificar a compreensão em leitura de estudantes de Ensino Fundamental, bem como explorar eventuais diferenças entre eles, no que se refere a gênero, ano escolar e idade. Participaram 240 alunos, de ambos os gêneros, entre 10 e 17 anos $(M=12,85 ; D P=1,60)$, do sexto ao nono ano de uma escola pública do interior de São Paulo. Um questionário de identificação e um texto com base na técnica de Cloze foram aplicados coletivamente aos escolares cujos pais ou responsáveis assinaram o Termo de Consentimento Livre e Esclarecido. Os resultados revelaram um nível básico de compreensão em leitura, entre os participantes, muito aquém do esperado para a sua escolarização. A pontuação das meninas foi significativamente mais alta, quando comparada à dos meninos. Houve também um incremento significativo das médias obtidas em função do ano escolar e das idades dos participantes.

Palavras-chave: leitura, Cloze, avaliação psicoeducacional
\end{abstract}

\section{Reading Comprehension for Students from the Third and Fourth Cycles of Basic Education}

\begin{abstract}
This study aimed at identifying reading comprehension of elementary school students and explore age, school year and gender related differences among the participants. The sample was composed of 240 students of both genders, with ages range between 10 and 17 years $(M=12.85 ; S D=1.60)$, from the sixth to the ninth school year of a public school in an inner city in the state of São Paulo. An identification questionnaire and a text based activity using Cloze technique were applied collectively to students whose parents signed the informed consent. Results showed basic level of reading comprehension, among participants, far behind the expectation for their school grade level. Girls scored significantly higher when compared to boys. There was a significant increase in mean values due to the school year and the participants' ages.

Keywords: reading, Cloze, psychoeducational assessment
\end{abstract}

\section{Comprensión de la Lectura en Estudiantes de Tercer y Cuarto Ciclo de Enseñanza Primaria}

\begin{abstract}
Resumen
Este estudio tuvo como objetivo identificar la comprensión de la lectura en estudiantes de Enseñanza Primaria, y también ver entre ellos las posibles diferencias, en relación a sexo, edad y curso escolar. Participaron 240 alumnos, de ambos sexos, entre 10 y 17 años $(M=12,85 ; S D=1,60)$, de sexto a noveno año de una escuela pública del interior de San Pablo. Un Cuestionario de Identificación y un texto basado en la técnica de Cloze fueron aplicados colectivamente a los escolares cuyos padres o responsables firmaron el formulario de consentimiento. Los resultados revelaron un nivel básico de comprensión de la lectura, pero mucho menos de lo esperado para su escolarización. El puntaje de las niñas fue significativamente mayor en comparación con el de los niños. También hubo un aumento significativo de las medias obtenidas en función del año escolar y edad de los participantes.

Palabras-clave: lectura, Cloze, evaluación psicoeducativa
\end{abstract}

Evidências revelam que a compreensão textual inclui vários processos cognitivos inter-relacionados, que envolvem desde os denominados processos básicos de leitura, como o reconhecimento e a extração do significado das palavras impressas, até cognitivos de alto nível, tais como a capacidade de realizar inferências, habilidades linguísticas gerais, habilidades de memória e conhecimento de mundo, que juntos contribuem para a construção de uma representação macroestrutural do texto e sua compreensão (Ayres, 1999; Cunha \& Santos, 2008; Ellis, 1995; Faria, 2011; Joly \& Piovezan, 2012; Monteiro \& Santos, 2013; Salles \& Parente, 2004; Randi, Grigorenko, \& Sternberg, 2009; Schreiber, 2009). Sob essa perspectiva, vários estudiosos consideram que leitores hábeis fazem inferências para relacionar ideias e obter informações que estão no texto de maneira implícita, são capazes de contextualizá-las e dispõem de estratégias que lhes possibilitem recorrer aos elementos intra, inter e extratextuais para chegar a sua compreensão crítica (Carpenter, Miyake, \& Just, 1995; Gomes \& Boruchovitch, 2013, 2014; Oliveira, Boruchovitch, \& Santos, 2009; Perfetti, 1992; Santos \& Oliveira, 2010).

Embora se espere que os alunos, ao finalizarem a primeira etapa formal de escolarização, sejam leitores hábeis e utilizem diferentes formas de linguagem para se comunicarem, a realidade observada é outra (Ministério da Educação - MEC, 1998; Mioto, 2014). No Brasil, independentemente da etapa de escolarização na qual 
o estudante se encontra, verifica-se que a ausência de estratégias adequadas de aprendizagem e bons hábitos de estudo estão associados a baixos índices de desempenho escolar (Alcará \& Santos, 2013; Joly \& Piovezan, 2012; Silva \& Witter, 2008; Watanabe, Cassetari, Santos, Lombard-Platet, \& Di Domenico, 2001). Mais precisamente, desempenhos aquém do esperado têm sido frequentes, por exemplo, em escolares da quarta (atual quinto ano) e oitava séries (atual nono ano) do Ensino Fundamental e do terceiro ano do Ensino Médio, tanto de escolas públicas, quanto de particulares (Instituto Nacional de Estudos e Pesquisas Educacionais Anísio Teixeira - INEP, 2007). Ademais, pesquisas realizadas com estudantes universitários mostram também que grande parcela deles lê pouco e tem dificuldade para ler e compreender textos (Alcará \& Santos, 2013; Calderón Ibañez \& Quijano Peñuela, 2010; Cunha \& Santos, 2006; Oliveira, 2011; Santos \& Silva, 2013). Constata-se, pois, que o problema da compreensão da leitura é sério, tem início nas séries iniciais do Ensino Fundamental e persiste ao longo do processo de escolarização. Avaliações educacionais nacionais quantificam sistematicamente a defasagem entre o que seria o desempenho desejável e o que é atingido por estudantes concluintes do Ensino Fundamental e Médio (INEP, 2007, 2012; Instituto Paulo Montenegro, 2013).

O descompasso entre as habilidades reais dos estudantes brasileiros e o que seria esperado ganha ainda mais relevância ao se considerar que um dos indicadores de desenvolvimento de um país é, justamente, o índice de alfabetismo. Para se ter uma ideia do cenário atual do país, segundo Cirilo Jr. (2013), o Instituto Brasileiro de Geografia e Estatística (IBGE) indicou a existência de 13,2 milhões de brasileiros com menos de quatro anos de escolaridade ou analfabetos funcionais, o que representa $8,7 \%$ da população de 15 anos ou mais.

O Instituto Paulo Montenegro e a Organização Não Governamental Ação Educativa trabalham com o Indicador de Analfabetismo Funcional (INAF), levando em conta habilidades práticas de leitura, escrita e matemática. A avaliação realizada envolve testes com tarefas de acesso cotidiano, tais como anúncios, instruções e notícias, classificando os entrevistados, com base no desempenho no teste, de acordo com o seu nível de alfabetismo que pode variar entre o analfabetismo, o alfabetismo nível rudimentar, o alfabetismo nível básico e o alfabetismo nível pleno. Pesquisas realizadas de 2001 a 2012 por essas instituições, com 2000 pessoas entre 15 e 64 anos, revelaram que, embora, nos últimos dez anos, tenha ocorrido uma redução do analfabetismo absoluto e da alfabetização rudimentar, bem como um incremento do nível básico de habilidades de leitura, escrita e matemática, apenas $25 \%$ delas dominam plenamente as habilidades de leitura e escrita. Além disso, $6 \%$ demonstraram um nível de habilidade muito abaixo, situando-se no nível rudimentar de alfabetismo, o que significa apenas a capacidade de localizar informações simples em enunciados de uma frase (Instituto Paulo Montenegro, 2013).

Constata-se ainda, por um lado, que o desempenho dos estudantes brasileiros melhorou nas avaliações internacionais, mas, por outro, fica evidente que a evolução é muito distante do que seria esperado. Dados de 2009 do Programa Internacional de Avaliação de Estudantes (PISA), uma prova internacional de compreensão de leitura e de outras competências vitais, aplicada a jovens com 15 anos já completos e que estejam frequentando pelo menos o sétimo ano em uma instituição formal de ensino, evidenciaram que o Brasil ficou na $53^{a}$ posição e obteve 412 pontos, pontuação abaixo da média do exame (500 pontos). Seu desempenho foi inferior ao de outros países da América Latina como o Chile $\left(44^{\circ}\right)$, Uruguai $\left(47^{\circ}\right)$, México $\left(48^{\circ}\right)$ e Colômbia $\left(52^{\circ}\right)$. No que concerne especificamente aos resultados relativos à proficiência em leitura, 49\% dos alunos brasileiros ficaram no nível 1 ou abaixo e, portanto, entre as piores classificações do exame. Cabe esclarecer que no PISA a proficiência em leitura pode ser classificada em oito escalas (abaixo nível 1b, nível 1b, nível 1a, nível 2, nível 3, nível 4, nível 5 e nível 6). De acordo com a Organização para a Cooperação e o Desenvolvimento Econômico (OCDE), pontuações abaixo do nível 2 são consideradas como baixo desempenho (Cunha, Suehiro, Oliveira, Pacanaro, \& Santos, 2009; Gomes \& Boruchovitch, 2009, 2013, 2014; INEP, 2012; OCDE, 2010; Okada, 2010; Santos \& Cunha, 2012).

Para além das implicações e críticas existentes em relação ao PISA, pesquisas nacionais, em menor escala, voltadas para identificar a compreensão da leitura em estudantes do Ensino Fundamental também mostram que eles estão aquém do nível de compreensão esperado para a etapa de escolarização na qual se encontram (Joly \& Nicolau, 2005; Oliveira, Boruchovitch, \& Santos, 2007; Oliveira et al., 2009; Suehiro \& Santos, 2009; Vendemiatto, 2007; entre outras). Joly e Nicolau (2005), por exemplo, verificaram que, dos 511 alunos de quarta série (atual quinto ano) do Ensino Fundamental por elas avaliados, $66 \%$ tinham dificuldade para compreender o que liam. 
De acordo com Oliveira, Boruchovitch e Santos (2007), essa defasagem e as sérias dificuldades na habilidade de leitura, apresentadas por muitos alunos do Ensino Fundamental, pode ser explicada pelo fato de que os estudantes que concluem esse estágio da educação formal, apesar de adquirirem a habilidade da leitura, não evidenciam a capacidade de abstrair as ideias mais relevantes do texto. Frequentemente, é possível observar que esses escolares realizam apenas decodificações simples, o que não denota o desenvolvimento de processos cognitivos de maior complexidade e compromete a compreensão do que foi lido. Apesar de a decodificação ser uma habilidade de base, sobre a qual se constroem as competências de nível mais elevado de tratamento do texto, a competência em leitura depende da combinação entre a decodificação e a atribuição de significados (Braibant, 1997; Faria, 2011; Joly \& Istome, 2008; Oliveira \& Capellini, 2013; Salles \& Parente, 2004; Sousa, 2005).

A técnica ou teste de Cloze é um dos instrumentos que tem sido utilizado na avaliação da compreensão em leitura. Baseia-se na natureza interativa do processo de compreensão e salienta a importância das pistas gramaticais e semânticas, bem como dos padrões de linguagem e do conhecimento prévio sobre o assunto para que a compreensão do material lido se torne possível (Ayres, 1999; Davis, Davis, Jacobson, \& Stah, 1989; Ellis, 1995; entre outros). A utilidade e os benefícios dessa técnica têm sido observados pelas inúmeras publicações acerca das possibilidades de seu emprego e pelas diferentes evidências de validade do instrumento. (Abraham \& Chapelle, 1992; Bensoussan, 1990; Centofanti, Ferreira, \& Del Tedesco, 1997; Cunha, 2009; Oliveira et al., 2007; Santos, 2005; Suehiro, 2013; Vicentelli, 1999). Algumas considerações sobre a técnica de Cloze são tecidas, brevemente, a seguir.

\section{A Compreensão de Leitura e a Técnica de Cloze: Breves Consi- derações e Evidências Empiricas}

Criada em 1953, a técnica ou teste de Cloze está entre os primeiros procedimentos sistemáticos utilizados na avaliação da habilidade de leitura, possibilitando, tanto sua avaliação e diagnóstico, quanto intervenção. Consiste em um texto do qual se omite todo quinto vocábulo, que é substituído por um traço de tamanho equivalente ao da palavra omitida. A tarefa do respondente é, após a leitura do texto, preencher as lacunas com a palavra que julgar mais adequada para completar seu sentido (Oliveira, 2011; Oliveira et al., 2009; Suehiro, 2013; Suehiro \& Santos, 2009; para citar alguns). É considerada como um procedimento simples, flexível, que não coloca intermediários entre o leitor e o texto, porque o texto é o próprio teste. Sua preparação segue regras que variam em função do objetivo para o qual o texto será utilizado (inteligibilidade, validade, diagnóstico ou intervenção). Os parâmetros mais frequentes na omissão sistemática das palavras consistem na retirada de todo quinto, sétimo ou décimo vocábulo, na supressão de uma categoria gramatical (adjetivos, substantivos, verbos, entre outras) ou ainda na eliminação aleatória de 20\% dos vocábulos do texto (Ashby-Davis, 1985; Bormuth, 1968; Santos, 2004).

Há, ainda, diferenças relativas à sua forma. De acordo com Santos (2004), o texto de Cloze é geralmente apresentado por escrito, sendo a palavra omitida substituída por um traço de tamanho sempre igual, tal como proposto por Taylor (1953), ou proporcional ao tamanho do vocábulo omitido, como sugerido por Bormuth (1968). Quanto à estruturação do texto, Bitar (1989) salienta que várias são as diversificações sofridas em relação à forma tradicional proposta por Taylor. Dentre as variações surgiram, o Cloze lexical, o Cloze gramatical, o Cloze de múltipla escolha e o Cloze cumulativo. No Cloze lexical, somente os itens lexicais do texto são omitidos, enquanto que, no gramatical, omitem-se todos os itens relacionais (palavras sem significado próprio e que servem para conectar outras palavras estruturando o texto sintaticamente), ou seja, preposições, conjunções, entre outros. No que se refere ao Cloze de múltipla escolha, são oferecidas diferentes alternativas para o preenchimento da lacuna. Já no Cloze cumulativo, há a omissão sistemática de uma única palavra, sendo esta substituída por uma outra sem sentido. Nessa variação, portanto, a tarefa do leitor é identificar a palavra sem sentido cada vez que ela aparece no texto.

Os resultados obtidos por Bitar (1989), ao comparar as diferentes variações da técnica, evidenciaram que o teste de Cloze convencional e o teste de atividades tradicionais se correlacionaram quando os textos empregados exigiam o uso da capacidade interpretativa do leitor, além da capacidade de compreensão. Sugeriram, ainda, que o Cloze convencional identificou o grau de maturidade do leitor com maior consistência. No entanto, a autora ressaltou a necessidade de se aprofundar a investigação para averiguar sua eficiência em relação ao teste de Cloze lexical. Já o teste de Cloze gramatical, segundo as análises, favoreceu o mascaramento das reais habilidades de leitura. Assim, na presente pesquisa optou-se pela utilização do Cloze convencional 
ou tradicional por se acreditar, tal como Bitar, que, ao omitir todo quinto vocábulo independentemente de sua classe gramatical ou significado, obtêm-se uma medida mais precisa da compreensão em leitura dos estudantes.

Sua correção pode ser realizada de forma literal ou sinônima (Bitar, 1989). A literal considera como acerto somente o preenchimento correto da palavra exata que foi omitida, respeitando sua grafia e acentuação. Já a sinônima, aceita um sinônimo da palavra omitida também como correto. Para tanto, deve-se utilizar um critério para o emprego dos sinônimos, tal como um dicionário conceituado de língua portuguesa, por exemplo. Tradicionalmente, a primeira forma é recomendada para efeitos de diagnóstico e de pesquisa, por evitar a subjetividade e facilitar sua correção (Bitar, 1989; Cunha \& Santos, 2014; Mota \& Santos, 2014).

Independentemente do critério adotado, diversos autores ressaltam a versatilidade da técnica de Cloze que, por seu de fácil manuseio, baixo custo, simplicidade e confiabilidade na avaliação do nível de compreensão de leitura, tem sido muito utilizada, desde o Ensino Fundamental até o Superior (Oliveira, 2011; Santos, Boruchovitch, \& Oliveira, 2009; Suehiro, 2013). Estudos como o de Suehiro (2013) mostram uma maior frequência de trabalhos, relativos à técnica de Cloze no período compreendido de 2002 a 2012, bem como que o público-alvo dessas pesquisas é composto, predominantemente, por estudantes do Ensino Fundamental. Todavia, evidenciou-se um número restrito de investigações que se tenham debruçado sobre a análise dos escolares do terceiro e do quarto ciclos. A maioria aponta baixos desempenhos em compreensão em leitura por parte de seus participantes (Oliveira et al., 2009; Santos, Suehiro \& Vendemiatto, 2009; Vendemiatto, 2007). O estudo de Vendemiatto (2007), realizado com 39 adolescentes em situação de risco, de quinta a oitava série (atuais sexto a nono anos) do Ensino Fundamental, revelou que a média de acertos dos participantes, em um texto elaborado para ser utilizado com crianças de etapas anteriores de escolarização, correspondeu ao nível intermediário de compreensão (instrucional). Ao lado disso, a autora não encontrou diferenças significativas entre as pontuações em compreensão de leitura e desenvolvimento perceptomotor em razão do gênero, série ou idade dos participantes.

Diferentemente do constatado por Vendemiatto, alguns estudos realizados com estudantes do terceiro e do quarto ciclos do Ensino Fundamental, assim como com etapas anteriores, identificaram diferenças significativas no desempenho de seus participantes em compreensão em leitura, quando se considera a idade, o gênero e o ano escolar, entre outras variáveis. De maneira geral, essas pesquisas evidenciam desempenhos significativamente superiores em meninas (Gomes \& Boruchovitch, 2014; Oliveira et al., 2007; Robbi, 2013; Santos, Lima, Silveira, Oliveira, \& Leme, 2010), estudantes de escola particular (Oliveira et al., 2007), mais velhos (Robbi, 2013; Santos et al., 2009) e nos anos escolares mais avançados (Mota \& Santos, 2014; Oliveira et al., 2007; Robbi, 2013; Santos et al., 2009). Investigações acerca da relação entre a compreensão de leitura e o desempenho escolar dos alunos de quinta a oitava séries (atuais sexto a nono ano), nas disciplinas de Português e Matemática, mostraram também que os estudantes em processo de aprendizagem, conforme categorizado pelos docentes, obtiveram médias mais baixas no teste de Cloze do que os que faziam as atividades dessas disciplinas, com e sem auxílio deles, respectivamente (Oliveira, Boruchovitch, \& Santos, 2008). Alunos que apresentaram melhores conceitos em Português (desempenho real) e uma autopercepção mais positiva (desempenho autopercebido) foram os que obtiveram as melhores pontuações em compreensão em leitura (Oliveira et al., 2009).

Pode, pois, constatar-se, pelos resultados provenientes das pesquisas nacionais, que o cenário é alarmante, caracterizado por alunos com um desempenho em leitura muito aquém do necessário para uma aprendizagem de qualidade, bem como que alguns fatores, como a idade, o gênero e o ano escolar, são importantes para o entendimento das diferenças na compreensão da leitura entre os estudantes. Sabe-se que a leitura constitui a principal via de acesso à aprendizagem de diferentes conteúdos veiculados no ensino formal, bem como que possibilita a assimilação dos valores da sociedade e contribui para a formação de indivíduos ativos (Gomes \& Boruchovitch, 2009; Gregoire \& Pérart, 1997; Lima, 2012), o que torna a situação mais crítica. Leitores com dificuldades na compreensão da leitura, como falhas no processo de decodificação, carências de vocabulário, leitura oral pobre, deficiência de integração das informações e de memória, falta de estratégias de aprendizagem adequadas e falta de motivação para a leitura, entre outras dificuldades, tendem a retroalimentar um círculo vicioso. Nele evitam as atividades de leitura, não alcançando a prática necessária para sua fluência. Consequentemente, seu envolvimento e nível de motivação para essas atividades tendem a diminuir, tornando-os gradativamente menos motivados a estudar (Boruchovitch, 2001; Lepper \& Henderlong, 2000; 
Lepper, Hederlong, \& Iyengar, 2005; Rufini, Bzuneck, \& Oliveira, 2011; Santos, 2004).

Assim, torna-se evidente a necessidade premente de que, não só se promova, de fato, a aprendizagem da leitura, desde o início da escolarização, já que o desempenho dos estudantes nas séries iniciais é um fator altamente preditivo de seu desempenho subsequente, mas também de que se avalie essa habilidade de forma adequada. Essa avaliação possibilitará a implementação de estratégias que possam suprir as lacunas detectadas nos diversos níveis de ensino (Gomes \& Boruchovitch, 2009; Guidetti \& Martineli, 2009; Istome \& Joly, 2010). Nesse sentido, a relevância da compreensão leitora para a aprendizagem e a importância do Ensino Fundamental, como um estágio de educação formal de base (Ministério da Educação - MEC, 2013; Suehiro, 2013), aliadas às poucas pesquisas que se detiveram à avaliação da compreensão em leitura no terceiro e quarto ciclos dessa etapa motivaram a realização do presente estudo. Avaliar a habilidade de compreensão em leitura de um grupo de estudantes do terceiro e do quarto ciclos do Ensino Fundamental, bem como identificar eventuais diferenças entre eles, no que se refere ao gênero, ano escolar e idade, constituíram-se, assim, nos seus objetivos.

\section{Método}

\section{Participantes}

Participaram deste estudo 240 estudantes, ambos os gêneros, entre 10 e 17 anos $(M=12,85 ; D P=$ $1,60)$, do sexto ao nono ano do Ensino Fundamental de uma escola pública do interior de São Paulo. Do total da amostra, 61 escolares $(25,4 \%)$ frequentavam o sexto ano, $61(25,4 \%)$ o sétimo, $55(22,9 \%)$ o oitavo e $63(26,3 \%)$ o nono ano, sendo a maioria do gênero masculino $(n=134 ; 55,8 \%)$. A seleção da amostra foi realizada por conveniência.

\section{Instrumentos}

Questões de identificação. Dados sobre os seguintes aspectos dos participantes: nome, idade, gênero e ano escolar que frequentam. O nome foi imediatamente substituído por um número de protocolo, conforme recomendação da legislação sobre a ética na pesquisa.

Teste de Cloze. Foi utilizado o texto intitulado "Coisas da Natureza" (Santos, 2005) com, aproximadamente, 250 vocábulos. Preparado segundo os padrões tradicionais do teste de Cloze, foi desenvolvido especialmente para avaliar a compreensão de leitura em alunos de quinta a oitava série (atuais sexto ao nono ano) do Ensino Fundamental, público para o qual apresenta evidências de validade. Santos (2005), por exemplo, identificou evidências de validade de critério para o texto. A autora constatou diferenças significativas no desempenho dos alunos das quatro séries por ela avaliadas (quinta, sexta, sétima e oitava séries), com um incremento das médias dos escores compatível com o avanço na escolarização. Da mesma forma, Oliveira et al. (2007) observaram escores significativamente mais altos em séries mais avançadas, estudantes mais velhos e de escolas particulares.

A pontuação máxima possível para o texto é 40 pontos, número correspondente à quantidade de lacunas nele contidas. Por ser a mais recomendada para fins de pesquisa, a correção foi realizada com base na proposta literal (Bitar, 1989), na qual se consideram corretas somente as respostas que contenham palavras idênticas às omitidas. Logo, os participantes receberam: zero quando deixavam a lacuna em branco, suas respostas eram sinônimas ou a frase permitia interpretação dupla do complemento e, um ponto, para cada acerto (palavras idênticas às omitidas). Adotou-se também a classificação de Bormuth (1968), que propõe níveis para interpretar a pontuação no Cloze. Assim, o primeiro corresponde ao nível de "frustração", que inclui leitores com até $44 \%$ de acertos, o segundo nível, o "instrucional", com $44,1 \%$ a $57 \%$ de acertos, referindo-se àqueles estudantes que demonstraram compreensão apenas suficiente para entender algumas ideias trazidas no texto e, nesse caso, precisam de ajuda externa para alcançarem a compreensão e, por último, o nível "independente", que abarca os leitores com desempenho satisfatório em compreensão em leitura.

\section{Procedimento}

A coleta de dados foi realizada por meio de respostas em lápis e papel, de acordo com o procedimento padrão da técnica de Cloze. Após a aprovação do projeto pelo Comitê de Ética (informação retirada para preservar o anonimato do autor), os instrumentos foram aplicados, coletivamente, em horário de aula previamente cedido pelo professor na escola, que autorizou a realização da pesquisa. Inicialmente, os estudantes, cujos pais assinaram o Termo de Consentimento Livre e Esclarecido e que concordaram em participar da pesquisa, preencheram as questões de identificação do sujeito e, em seguida, foram solicitados a lerem o texto que lhes foi entregue e a preencherem as lacunas com 
as palavras que melhor se adequassem ao contexto e completassem seu sentido. Todos os estudantes foram submetidos ao mesmo texto em Cloze que, conforme já relatado, foi construído e validado especialmente para alunos da quinta à oitava série do Ensino Fundamental. A aplicação durou 20 minutos.

\section{Resultados}

Considerando-se os objetivos do estudo, foram utilizadas provas de estatística descritiva e inferencial não paramétricas, visto que o resultado da prova de Kolmogorov-Smirnov Z para a análise da curva da pontuação dos escores em compreensão em leitura permitiu recusar a hipótese de normalidade (Kolmogorov-Smirnov $Z=0,17 ; p<0,001)$. A pontuação total média obtida pelos estudantes avaliados no Cloze foi de 19,11 acertos $(D P=8,17)$, de um total de 40 possíveis. A pontuação mínima obtida foi zero e a máxima, 32 acertos.

Dos 240 escolares que participaram do estudo, 65 $(27,1 \%)$ tiveram o seu desempenho em compreensão de leitura classificado no nível de frustração, de acordo com os critérios de Bormuth (1968). Esse resultado indica que tais estudantes não abstraíram de forma satisfatória a informação lida (pontuação de até 44\% de acertos - até 17,6 pontos dos 40 possíveis). Outros 82 $(34,2 \%)$ participantes foram classificados com um nível de compreensão em leitura instrucional e, portanto, indicativo de que eles demonstraram uma habilidade apenas suficiente para o entendimento do texto empregado (de $44,1 \%$ a $57 \%$ de acertos - de 17,61 a 22,80 pontos dos 40 acertos possíveis).

Buscou-se, ainda, verificar se as pontuações no Cloze sofreriam alterações ao se considerarem variáveis como o gênero, o ano escolar e a idade dos estudantes. A Tabela 1 traz as diferenças relativas ao gênero dos participantes do estudo.

Como pode ser observado na Tabela 1, as médias dos ranks das meninas foram significativamente mais altas, quando comparadas às dos meninos $(z=4,33)$. Com o intuito de verificar se haveria diferença entre os estudantes ao se considerar o ano escolar por eles frequentado, recorreu-se ao teste para amostras independentes para mais de duas condições - Kruskal-Wallis. A análise evidenciou diferença significativa entre o desempenho em compreensão em leitura dos discentes $([H(3)=42,85 ; p<0,001])$, bem como que a média dos ranks obtida pelos escolares do nono ano foi maior que a dos de oitavo, sétimo e sexto, respectivamente. Esses dados podem ser visualizados na Tabela 2.

Para verificar se haveria diferença na compreensão em leitura também em relação à idade, recorreu-se a análise dos seus extremos. Para tanto, agrupou-se essa variável em três grupos, quais sejam, até 12 anos; 13 anos e 14 ou mais anos. Foi usado o teste Mann-Whitney para a comparação entre o grupo constituído pelos alunos mais novos e o formado pelos escolares mais velhos. Verificou-se que os estudantes mais velhos obtiveram uma média por ranks de acertos significativamente superior à dos mais novos $(z=-2,58)$, tal como pode ser observado na Tabela 3.

Tabela 1

Pontuações Obtidas em Razão do Gênero

\begin{tabular}{lccccc}
\hline Cloze & Gênero & $N$ & Média dos ranks & U & $p$ \\
\cline { 2 - 6 } Coisas da natureza & masculino & 133 & 102,75 & 9,34 & $<0,001$ \\
& feminino & 106 & 141,64 & & \\
\hline
\end{tabular}

Tabela 2

Comparação entre as Pontuações Obtidas por Ano escolar, pela Prova Kruskal-Wallis

\begin{tabular}{lccccc}
\hline Cloze & Anos Escolares & $N$ & Média dos ranks & $\chi 2(\mathrm{gl}=3)$ & $p$ \\
\cline { 2 - 6 } Coisas da natureza & Sexto & 61 & 93,75 & 42,85 & $<0,001$ \\
& Sétimo & 61 & 98,42 & & \\
& Oitavo & 54 & 120,28 & \\
& Nono & 63 & 166,07 & \\
\hline
\end{tabular}


Tais resultados indicam, portanto, um incremento na compreensão em leitura dos estudantes em função do seu desenvolvimento maturacional.

\section{Discussão}

A literatura aponta, frequentemente, que muitos estudantes que concluem o Ensino Fundamental não desenvolveram processos cognitivos de maior complexidade necessários para a capacidade de abstrair as ideias mais relevantes do texto, o que não assegura a compreensão do que foi lido (Faria, 2011; Joly \& Istome, 2008; Oliveira \& Capellini, 2013; Salles \& Parente, 2004; Sousa, 2005). Os resultados aqui obtidos caminham nessa direção, ao revelarem que os escolares avaliados alcançaram uma pontuação média ligeiramente inferior ao ponto médio do instrumento, que seria de 20 acertos, e estão, lamentavelmente, abaixo da expectativa para a sua etapa de escolarização.

O dado, ora obtido, pode ser melhor compreendido, ao se verificar que, dos 240 estudantes que participaram do estudo, 147 (61,3\%) e, portanto, mais da metade da amostra teve o seu desempenho em compreensão de leitura classificado nos níveis de frustração e instrucional, de acordo com os critérios de Bormuth (1968).Tais achados são coerentes com os veiculados por outras investigações com estudantes dessa etapa de escolarização, que identificaram uma capacidade de leitura, no mínimo básica, sem criticidade nem criatividade entre eles (INEP, 2007, 2012; Oliveira et al., 2007, 2008; PISA, 2009; Santos et al., 2009; Vendemiatto, 2007).

Em consonância com os resultados de Oliveira et al. (2007), com estudantes do Ensino fundamental II, Santos et al. (2010) e Robbi (2013), por exemplo, com estudantes de etapas anteriores, os meninos tenderam a apresentar mais problemas de compreensão em leitura. Tal como também emergiu na literatura, houve, na amostra estudada, uma tendência a um aumento progressivo na quantidade de acertos no Cloze, com o avançar da escolaridade (Oliveira et al., 2007; Santos et al., 2009). A média por ranks obtida pelos escolares do nono ano foi maior que a apresentada pelos de oitavo, sétimo e sexto, respectivamente.

Do mesmo modo, constatou-se um incremento na compreensão em leitura dos estudantes em função do seu desenvolvimento maturacional, uma vez que os escolares mais velhos obtiveram uma pontuação média, por ranks, superior de acertos. Esses resultados, todavia, divergem dos obtidos por Vendemiatto (2007) e Santos et al. (2009) que, ao avaliarem adolescentes em situação de risco, mesmo que com um texto estruturado para uma etapa de escolarização anterior a de suas amostras, não encontraram diferença estatisticamente significativa no desempenho em leitura em razão da idade. Essas divergências apontam a necessidade de que a variável idade seja mais explorada em pesquisas futuras relativas à compreensão leitora de estudantes do terceiro e quarto ciclos, já que, no caso de etapas de escolarização anteriores aqui selecionada, o aspecto maturacional esperado em razão da idade e da progressão escolar tem sido frequentemente observado (Mota \& Santos, 2014; Robbi, 2013; Santos et al., 2010; entre outros). Essas novas investigações poderiam, inclusive, comparar o desempenho de estudantes em situações mais favoráveis para a aprendizagem com àqueles em risco, de forma a se melhor aquilatar o seu impacto e nortear ações preventivas.

Os dados obtidos nesta pesquisa, em conjunto com a literatura da área (Cunha et al., 2009; Gomes \& Boruchovitch, 2009, 2013; INEP, 2012; Mioto, 2014; Santos \& Cunha, 2012), são preocupantes na medida em que desvelam que, embora se tenha verificado um incremento do nível básico de habilidades de leitura, escrita e Matemática no Brasil, nos últimos dez anos (Instituto Paulo Montenegro, 2013), esse aumento ainda parece estar bastante distante da qualidade necessária. Esse descompasso tem impactos psicoeducacionais importantes, sobretudo para o aluno, já que os resultados aqui encontrados podem gerar problemas motivacionais, emocionais, disciplinares e dificuldades na aprendizagem dos conteúdos veiculados ao longo dos anos, em cada etapa de escolarização. Ademais, estudantes do gênero masculino devem também ser alvos de maiores

Tabela 3

Pontuações Obtidas por Estudantes mais Novos e mais Velhos $(N=189)$

\begin{tabular}{lccccc}
\hline Cloze & Extremos de idade & $N$ & Média dos ranks & U & $p$ \\
\cline { 2 - 6 } Coisas da natureza & Até 12 anos & 105 & 85,83 & 3447 & 0,010 \\
& 14 anos ou mais & 84 & 106,46 & & \\
\hline
\end{tabular}

Psico-USF, Bragança Paulista, v. 21, n. 3, p. 561-572, set./dez. 2016 
esforços educacionais, por apresentarem tendência a maiores problemas de compreensão da leitura, quando comparados aos do gênero feminino. No que concerne ao sistema de ensino brasileiro, os problemas se acumulam e ganham proporções cada vez maiores, à medida que envolvem outros atores, tais como os professores. Nesse ciclo vicioso, a sociedade passa a contar, cada vez mais, com pessoas acríticas e que deixam de exercer plenamente sua condição de cidadãs.

Há que se pensar, por um lado, em programas e estratégias pedagógicas que possibilitem, não apenas uma aproximação, mas um maior envolvimento dos estudantes com a leitura. Essa aproximação precisa ser estimulada de modo que os interesses pelo assunto e os conhecimentos prévios dos alunos sejam contemplados e possibilitem que a leitura seja tida como uma atividade prazerosa e não como uma obrigação. Por outro, impera a necessidade de uma política educacional mais coerente com a aprendizagem de qualidade desejada por todos os brasileiros e que, de acordo com os estudos da área, ainda parece uma meta bastante distante.

Acredita-se que o presente estudo tenha gerado dados importantes sobre a habilidade de leitura dos estudantes avaliados, especialmente, porque pesquisas com escolares do terceiro e quarto ciclos do Ensino Fundamental não têm sido abundantes. Destarte, novas investigações com esses segmentos de escolarização são extremamente relevantes. Espera-se que essas futuras investigações venham não apenas diminuir as limitações deste estudo, utilizando amostras maiores (com o objetivo de obter estimativas de efeito mais precisas e maior poder), de instituições de natureza jurídica e regiões diferentes, mas que associem outras variáveis tão importantes para a aprendizagem quanto a leitura, como, por exemplo, a motivação para ler e aprender, bem como o uso de estratégias de leitura, entre outras. Ao considerar essas sugestões, essas pesquisas poderão ampliar os conhecimentos na área e possibilitar o desenvolvimento de estratégias de intervenção sobre os problemas detectados, contribuindo, assim, para o favorecimento do processo de aprendizagem desses escolares, num enfoque que possa ultrapassar o caráter meramente remediativo.

\section{Referências}

Abraham, R. G., \& Chapelle, C. A. (1992). The meaning of Cloze test scores: An item difficulty perspective. The Modern Language Journal, 76(4), 468-479. doi: 10.1111/j.1540-4781.1992.tb05394.x
Alcará, A. R., \& Santos, A. A. A. (2013). Compreensão de leitura, estratégias de aprendizagem e motivação em universitários. Psico, 44(3), 411-420.

Ashby-Davis, C. (1985). Cloze and reading comprehension: A qualitative analysis and critique. Journal of Reading, 28(7), 585-589.

Ayres, C. R. (1999). O papel do conhecimento prévio na relação leitura e compreensão. Signo, 24(37), 71-85.

Bensoussan, M. (1990). Redundancy and the cohesion Cloze. Journal of Research in Reading, 13(1), 18-37. doi: 10.1111/j.1467-9817.1990.tb00320.x

Bitar, M. L. (1989). Eficiência dos instrumentos de avaliação em leitura (Dissertação de mestrado). Pontifícia Universidade Católica de São Paulo, São Paulo-SP.

Bormuth, J. R. (1968). Cloze Test readability: Criterion references scores. Journal of Educational Measurement, 5(3), 189-196. doi: 10.1111/j.1745-3984.1968. tb00625.x

Boruchovitch, E. (2001). Algumas estratégias de compreensão em leitura de alunos do ensino fundamental. Psicologia Escolar e Educacional, 5(1), 19-25. doi: 10.1590/S1413-85572001000100003

Braibant, J. (1997). A decodificação e a compreensão: Dois componentes essenciais da leitura no $2^{\circ}$ ano primário. Em J. Grégoire, \& B. Piérart (Eds.), Avaliação dos problemas de leitura: os novos modelos teóricos e suas implicações diagnósticas (pp.167-187). Porto Alegre: Artes Médicas.

Calderón Ibañez, A., \& Quijano Peñuela, J. (2010). Características de comprensión lectora em estudiantes universitários. Jurídicas, 7(2), 123-151.

Carpenter, P. A., Miyake, A., \& Just, M. A. (1995). Language comprehension: Sentence and discourse processing. Annual Reviews Psychology, 46, 91-120. doi: 10.1146/annurev.ps.46.020195.000515

Centofanti, E. M., Ferreira, S. M., \& Del Tedesco, T. (1997). Compreensão da leitura por universitários de psicologia. Em G. P. Witer (Eds.), Leitura e universidade (pp. 33-60). Campinas: Alínea.

Cirilo Jr., (2013). IBGE: analfabetismo cresce pela primeira vez desde 1998. Recuperado de http:// noticias.terra.com.br/educacao/ibge-analfabetismo-cresce-pela-primeira-vez-desde-1998,e5e1e55448c51410VgnVCM3000009acceb0aRCRD. html. 
Cunha, N. B. (2009). Pesquisas com o teste de Cloze no Brasil. Em A. A. A. Santos, E. Boruchovitch \& K. L. Oliveira (Eds.), Cloze: um instrumento de diagnóstico e intervenção (pp. 79-117). São Paulo: Casa do Psicólogo.

Cunha, N. B., \& Santos, A. A. A. (2006). Relação entre a compreensão da leitura e a produção escrita em universitários. Psicologia: Reflexão e Crítica, 19(2), 237-245. doi: 10.1590/S0102-79722006000200009

Cunha, N. B., \& Santos, A. A. A. (2008). Habilidades linguísticas no ensino fundamental em escolas públicas e particulares. Psic (São Paulo), 9(1), 35-44.

Cunha, N. B., \& Santos, A. A. A. (2014). Estudo de validade do questionário de avaliação de consciência metatextual. Revista Psicologia: Teoria e Prática, 16(1), $141-154$.

Cunha, N. B., Suehiro, A. C. B., Oliveira, E. Z., Pacanaro, S. V., \& Santos, A. A. A. (2009). Produção científica da avaliação da leitura no contexto escolar. Psico, 40(1), 17-23.

Davis, R. L., Davis, C. E., Jacobson, M. G., \& Stahl, S. A. (1989). Prior knowledge and difficult vocabulary in the comprehension of unfamiliar text. Reading Research Quaterly, 24(11), 27-41.

Ellis, A. W. (1995). Leitura, escrita e dislexia: uma análise cognitiva. Tradução Dayse Batista ( $2^{\mathrm{a}}$ ed.), Porto Alegre: Artes Médicas.

Faria, E. L. B. (2011). Estratégias de compreensão da leitura: Perspectivas teóricas. Mal-Estar e Sociedade, IV (6), 83-98.

Gomes, M. A. M., \& Boruchovitch, E. (2009). Proficiência em leitura: Um panorama da situação. Em A. A. A. dos Santos, E. Boruchovitch \& K. L. de Oliveira (Eds.), Cloze: um instrumento de diagnóstico e intervenção (pp. 23-46). São Paulo: Casa do Psicólogo.

Gomes, M. A. M., \& Boruchovitch, E. (2013). Leitura e compreensão: Contribuição da psicologia cognitiva e da teoria do processamento da informação. Em A. Spinillo; M. M. P. E. da Mota. (Eds.), Compreensão de Texto (pp. 111-132, 1ed). São Paulo: Cada do Psicólogo.

Gomes, M. A. M., \& Boruchovitch, E. (2014). Motivation to read and reading comprehension of Brazilian students. Educatio Siglo XXI, 32(2), 119138. doi: org/10.6018/j/202191
Gregoire, J., \& Piérart, B. (1997). Avaliação problemas de leitura: Os novos modelos teóricos e suas implicações. Tradução Maria Regina Borges Osório. Porto Alegre: Artes Médicas.

Guidetti, A. A., \& Martinelli, S. C. (2009). Desempenho em leitura e suas relações com o contexto familiar. Em A. A. A. Santos, E. Boruchovitch \& K. L. Oliveira (Eds), Cloze: um instrumento de diagnóstico e intervenção (pp. 283-309). São Paulo: Casa do Psicólogo.

Instituto Nacional de Estudos e Pesquisas Educacionais Anísio Teixeira - INEP (2007). Qualidade da educação: Uma nova leitura do desempenho dos estudantes de $4^{a}$ série do Ensino Fundamental. Recuperado de http://www.inep.gov.br

Instituto Nacional de Estudos e Pesquisas Educacionais Anísio Teixeira - INEP (2012). Programa internacional de avaliação de alunos (PISA): Resultados nacionais PIS A 2009. Brasília: Instituto Nacional de Estudos e Pesquisas Educacionais.

Instituto Paulo Montenegro (2013). Instituto Paulo Montenegro e Ação Educativa mostram evolução do alfabetismo funcional na última década. Recuperado de http://www.ipm.org.br/ipmb_pagina. php?mpg $=4.02 .01 .00 .00 \&$ ver $=$ por.

Istome, A. C., \& Joly, M. C. R. A. (2010). Estudo correlacional do teste dinâmico de leitura com WISC III. Psicologia: Teoria e Prática, 12(1), 43-58.

Joly, M. C. R. A., \& Istome, A. C. (2008). Compreensão em leitura e capacidade cognitiva: Estudo de validade do teste Cloze Básico - MAR. Psic (São Paulo), 9(2), 219-228.

Joly, M. C. R. A, \& Nicolau, A. F. (2005). Avaliação de compreensão em leitura usando cloze na $4^{a}$ série. Temas sobre desenvolvimento, 14(83/84), 14-19.

Joly, M. C. R. A., \& Piovezan, N. M. (2012). Avaliação do programa informatizado de leitura estratégica para estudantes do ensino fundamental. Paidéia, 22(51), 83-90. doi: 10.1590/S0103-863X2012000100010

Lepper, M. R., \& Henderlong, J. (2000). Turning “play" into "work" and "work" into "play": 25 years of research on intrinsic versus extrinsic motivation. Em C. Sansone \& J. M. Harackiewicz (Eds.), Intrinsic and extrinsic motivation: the search for optimal motivation and performance (pp. 257-307). San Diego: Academic Press. 
Lepper, M. R., Henderlong, J., \& Iyengar, S. S. (2005). Intrinsic and extrinsic motivational orientations in the classroom: Age differences and academic correlates. Journal of Educational Psychology, 97(2), 184-196. doi: 10.1037/0022-0663.97.2.184

Lima, L. B. V. (2012). Depressão infantil, compreensão de leitura e escrita: Um estudo com crianças do ensino fundamental (Dissertação de mestrado). Programa de Pós-Graduação Stricto Sensu em Psicologia, Universidade São Francisco, Itatiba, São Paulo.

Ministério da Educação - MEC (1998). Parâmetros curriculares nacionais: Terceiro e quarto ciclos do Ensino Fundamental: Língua portuguesa. Recuperado de http://portal.mec.gov.br/seb/arquivos/ pdf/portugues.pdf

Ministério da Educação - MEC (2013). Pacto Nacional pela Alfabetização na Idade Certa. Recuperado de http://pacto.mec.gov.br/o-pacto.

Mioto, R. (2014). Abismo na educação ainda separa Brasil e paises ricos. Recuperado de http://www1.folha.uol. com.br/fsp/mercado/170320-abismo-na-educacao-ainda-separa-brasil-e-paises-ricos.shtml

Monteiro, R. M., \& Santos, A. A. A. (2013). Recursos familiares e desempenho de crianças em compreensão de leitura. Psico, 44(2), 273-279.

Mota, M. M. P. E., \& Santos, A. A. A. (2014). O Cloze como instrumento de avaliação de leitura nas séries iniciais. Psicologia Escolar e Educacional, 18(1), 135142. doi: 10.1590/S1413-85572014000100014

Organização para a Cooperação e o Desenvolvimento Econômico - OCDE (2010). What students know and can do: student performance Reading, Mathematics and Science. (Pisa 2009 Results, Volume I). Recuperado de http://www.oecd-ilibrary.org/education/ pisa-2009-results-what-students-know-and-can-do_9789264091450-en

Okada, A. (2010). Pisa 2009: Xangai, na China, lidera ranking de leitura entre estudantes; Brasil está em 53․ UOL Educação. Recuperado de http:// educacao.uol.com.br/noticias /2010/12/07/ pisa-2009-china-lidera-ranking-de-leitura-brasil-esta-esta-em-53.htm

Oliveira, A. M., \& Capellini, S. A. (2013). Compreensão leitora de palavras e frases: elaboração de procedimento avaliativo. Psicologia em Estudo, 18(2), 293-301. doi: 10.1590/S1413-73722013000200010
Oliveira, K. L. (2011). Considerações acerca da compreensão em leitura no ensino superior. Psicologia: Ciência e Profissão, 31(4), 690-701. doi: /10.1590/ S1414-98932011000400003

Oliveira, K. L., Boruchovitch, E., \& Santos, A. A. A. (2007). Compreensão de leitura em alunos de sétima e oitava séries do ensino fundamental. Psicologia Escolar e Educacional, 11(1), 41-49. doi: 10.1590/ S1413-85572007000100005

Oliveira, K. L., Boruchovitch, E., \& Santos, A. A. A. (2008). Leitura e desempenho escolar em português e matemática no ensino fundamental. Paidéia, 18(41), 531-540. doi: 10.1590/ S0103-863X2008000300009

Oliveira, K. L., Boruchovitch, E., \& Santos, A. A. A. (2009). Leitura e desempenho escolar em alunos do Ensino Fundamental. Em A. A. A. Santos, E. Boruchovitch \& K. L. Oliveira (Eds.), O Cloze como instrumento de diagnóstico e intervenção (pp. 149-164). São Paulo: Casa do Psicólogo.

Perfetti, C. A. A. (1992). Capacidade para a leitura. Em R. J. Sternberg (Eds.), As capacidades intelectuais bumanas (pp.72-76). Tradução de Dayse Batista. Porto Alegre: Artes Médicas.

Randi, J., Grigorenko, E. L., \& Sternberg, R. J. (2009). Revisiting definitions of reading comprehension: Just what is reading comprehension anyway? Em $\mathrm{S}$. E. Israel, C. C. Block, K. L. Bauserman \& K. Kinnucan-Welsch (Eds.), Metacognition in literacy learning: Theory, assessment, instruction and professional development (pp. 19-39). Mahwah, NJ: Lawrence Erlbaum.

Robbi, D. M.P. (2013). Compreensão leitora e desempenho em matemática e escrita: Estudo com alunos do Ensino Fundamental 1 (Dissertação de mestrado). Programa de Pós-Graduação Stricto Sensu em Psicologia, Universidade São Francisco, Itatiba, São Paulo.

Rufini, S. E., Bzuneck, J. A., \& Oliveira, K. L. (2011). Estudo de validação de uma medida de avaliação da motivação para alunos do ensino fundamental. Psico-USF, 16(1), 1-9. doi: 10.1590/ S1413-82712011000100002

Salles, J. F., \& Parente, M. A. M. P. (2004). Compreensão textual em alunos de segunda $\mathrm{e}$ terceiras séries: Uma abordagem cognitiva. Estudos de Psicologia (Natal), 9(1), 71-80. doi: 10.1590/ S1413-294X2004000100009 
Santos, A. A. A. (2004). O Cloze como técnica de diagnóstico e remediação da compreensão em leitura. Interação em Psicologia, 8(2), 217-226. doi: 10.5380/ psi.v8i2.3257

Santos, A. A. A. (2005). O teste de Cloze como instrumento de diagnóstico e de desenvolvimento da compreensão em leitura (Relatório técnico de bolsista produtividade enviado ao CNPq). Universidade São Francisco, Itatiba, São Paulo.

Santos, A. A. A., Boruchovitch, E., \& Oliveira, K. L. (2009) (Eds.). Cloze: Um instrumento de diagnóstico e intervenção. São Paulo: Casa do Psicólogo.

Santos, A. A. A., \& Cunha, N. B. (2012). Consciência metatextual: Evidências de validade para instrumento de medida. Psico-USF, 17(2), 233-241. doi: 10.1590/S1413-82712012000200007

Santos, A. A. A., Lima, T. H., Silveira, F. J. Oliveira, N. R. P., \& Leme, E. M. (2010). Relação entre a compreensão de leitura e a consciência fonológica. Em L. S. Almeida, B. D. Silva \& S. Caires. (Eds.), Actas do I Seminário Internacional "Contributos da Psicologia em Contextos Educativos". Braga: CIEd - Centro de Investigação em Educação, Instituto de Educação, Universidade do Minho.

Santos, A. A. A., \& Oliveira, E. Z. (2010). Avaliação e desenvolvimento da compreensão em leitura no ensino fundamental. Psico-USF, 15(1), 93-102. doi: 10.1590/S1413-82712010000100009

Santos, A. A. A., Suehiro, A. C. B., \& Vendemiatto, B. C. (2009). Inteligencia y comprensión en lectura de adolescentes en situación de riesgo social. Paradigma (Maracay), 30(2), 117-127.

Santos, N. L., \& Silva, S. L. C. (2013). A compreensão leitora de alunos de cursos técnicos e superiores. Tear: Revista de Educação Ciência e Tecnologia, 2(1), 01-12.

Schreiber, F. J. (2009). Metacognition and self-regulation in literacy. In S. E. Israel, C. C. Block, K. L. Bauserman \& K. Kinnucan-Welsch (Eds.), Metacognition in literacy learning: Theory, assessment, instruction and professional development (pp. 215-240). Mahwah, NJ: Lawrence Erlbaum.

Silva, E. M. T. \& Witter, G. (2008). Compreensão de texto e desempenho acadêmico em estudantes de psicologia. Estudos de Psicologia (Campinas), 25(3), 395-403. doi: 10.1590/S0103-166X2008000300008

Sousa, E. O. (2005). Habilidades metassintáticas e aprendizagem da leitura: Estudo com crianças da $1^{a}$ série do Ensino Fundamental (Tese de doutorado). Pontifícia Universidade Católica de São Paulo, São Paulo.

Suehiro, A. C. B. (2013). Produção científica sobre o teste de Cloze. Psicologia Escolar e Educacional, 17(2), 223-232. doi: 10.1590/S1413-85572013000200004

Suehiro, A. C. B., \& Santos, A. A. A. (2009). O teste de Cloze e o desenvolvimento perceptomotor no início da escolarização. Em A. A. A. Santos, E. Boruchovitch \& K. L. Oliveira (Eds.), Cloze: um instrumento de diagnóstico e intervenção (pp. 187-225). São Paulo: Casa do Psicólogo.

Taylor, W. L. (1953). Cloze procedure: A new tool for measuring readability. Journalism Quarterly, 30, 415-433.

Vendemiatto, B. C. (2007). Medidas de habilidades cognitiva e visomotora: evidências de validade do Bender-SPG (Dissertação de mestrado). Universidade São Francisco, Itatiba, São Paulo.

Vicentelli, H. (1999). Problemática de la lectura en estudiantes universitarios. Psicologia Escolar e Educacional, 3(3), 195-202.

Watanabe, O. M., Cassetari, L., Santos, M. L. M., Lombard-Platet, V. L. V., \& Di Domenico, V. G. C. (2001). Um levantamento dos hábitos de estudo dos alunos do curso de psicologia de centro universitário FMU (Faculdades Metropolitanas Unidas). Psikhê, 6, 60-71.

Recebido 23/03/2015

Reformulado 15/07/2015 Aceito 27/08/2015 
Sobre as autoras:

Adriana Cristina Boulhoça Suehiro é doutora em Psicologia pelo Programa de Pós-Graduação Stricto Sensu em Psicologia da Universidade São Francisco e docente da Universidade Federal do Recôncavo da Bahia - Santo Antônio de Jesus. Possui pós-doutorado em Educação pela Unicamp, é líder do Laboratório de Instrumentação e Avaliação Psicológica da Universidade Federal do Recôncavo da Bahia (LABIAP) e membro do Grupo de Estudos e Pesquisas em Psicopedagogia (GEPESP-UNICAMP).

E-mail:dricbs@yahoo.com.br

Evely Boruchovitch é psicóloga, $\mathrm{PhD}$ em Educação pela University of Southern Califórnia, professora titular do Departamento de Psicologia Educacional e do Programa de Pós-graduação em Educação da Faculdade de Educação da Universidade Estadual de Campinas (UNICAMP), membro do Grupo de Estudos e Pesquisas em Psicopedagogia (GEPESP-UNICAMP) e bolsista produtividade do CNPq.

E-mail: evely@unicamp.br

Contato com as autoras:

Universidade Federal do Recôncavo da Bahia (UFRB) - Centro de Ciências da Saúde

Av. Carlos Amaral, 1015 - Cajueiro

CEP: 44570-000

Santo Antônio de Jesus - Bahia, Brasil

E-mail:dricbs@yahoo.com.br

Telefone: (75) 3632-4629 